\title{
A MODEL OF THE GALACTIC DISK WITH A CENTRAL HOLE
}

\author{
J.R.D. LÉPINE ${ }^{1}$, P. LEROY ${ }^{2}$ \\ 1)Instituto Astronômico e Geofísico, University of São Paulo, \\ 2)Departamento de Física, Universidade Federal de Minas Gerais, \\ Brazil
}

\section{Introduction}

We present a model aimed to reproduce the infrared brigtness distribution of the Galaxy, as observed by the COBE-DIRBE experiment, and by the Japanese balloon flights (eg. Hayakawa et al., 1981). Based on the disk brightness profiles of a sample of spiral galaxies, and on the observed brigthness distribution of bars, we adopted new mathematical expressions to represent each component (bulge, bar, disk, spiral arms).

\section{The basic model}

The description of the stellar populations (density of stars as a function of spectral type and luminosity class) and of the interstellar extinction distribution, is the same of Ortiz and Lépine, 1993. We used the Monte Carlo method to generate spatial distributions of stars, according to the adopted mathematical expressions for each component of the Galaxy. The fluxes of the stars were then added in a grid in galactic coordinates, taking into account their distances from the Sun and the interstellar extinction. The main components of the Galaxy were described as follows. The density of the bulge, for each spectral type $s$, is given by:

$$
n(r, s)=k(s) \frac{a^{4}}{r(r+a)^{3}}
$$


Where $a$ is a scale-size. For the disk, we use a difference of two radially decreasing exponentials:

$$
n_{i}(r, z, s)=c_{i}(s)\left(e^{\frac{-r}{\alpha}}-e^{\frac{-2 r}{\alpha}}\right) \operatorname{sech}^{2}\left(\frac{z}{\beta_{i}}\right)
$$

where $\alpha$ is the scale-length and $\beta$ the scale-height. We use two such disks, with scale-heights $100 \mathrm{pc}$ and $400 \mathrm{pc}$ respectively. We have tested these expressions on many galaxies, by integrating equations (1) and (2) along the line-of-sight. The spiral arms have their radius given by:

$$
\rho_{s p i}=q . \exp \left[\left(\theta-\theta_{0}\right) \tan (i)\right]
$$

in the interval $\rho_{1}<\rho_{s p i}<\rho_{2}$, with a Gaussian distribution of stellar density around the radius $\rho_{s p i}$ :

$$
n(\rho, \theta, z)=n_{g} \exp \left[-\left(\rho-\rho_{s p i}\right)^{2} / \gamma^{2}\right] \exp \left(-z / \beta_{1}\right)
$$

The internal starting point of the arms is $\rho_{1}=2.5 \mathrm{kpc}$. The bar is described as a Gaussian distribution of stars similar to the arms, extending $2.5 \mathrm{kpc}$ each side of the galactic center. Following the model of Amaral and Lépine (1997), we consider 4 arms; two of them start at the extremities of the bar.

\section{Results}

We obtained a good fit of the infrared brightness distribution of the Galaxy, taken from the COBE-DIRBE experiments and the Japanese baloon flights. The main conclusions are the following: 1)There is no reason for the disk being exponential down to the center of the Galaxy, in the region of the bulge. A good fit of external galaxies and of the Galaxy is obtained with Equation (2), which corresponds to a distribution with a hole in the center of the disk. 2)Bars in external galaxies are not well represented by triaxial spheroids. A bar is a flat elongated structure, distinct from the inner bulge, which is a spheroid. 3)The bar of the Galaxy, seen almost edge-on, is consistent with the 4 arms spiral structure described by Amaral and Lépine (1997). Two of the arms start at the extremeties of the bar. 4) The Galaxy has no ring. The maximum at $l=30^{\circ}$ is a tangential direction to one of the spiral arms. 5)The maximum of the rotation curve at $300 \mathrm{pc}$ is due to circular rotation around the inner bulge.

\section{References}

Amaral, L.H., Lépine, J.R.D., 1997, MNRAS,286,885

Hayakawa,S., Matsumoto, T., Murakami, H., Uyama, K., Thomas, J.A., Yamagami, T., $1981, A \& A, 100,116$

Ortiz, R., Lépine, J.R.D., 1993, $A \mho \exists A, \mathbf{2 7 9 , 9 0}$ 